\title{
FCLA chemiluminescence from sonodynamic action in vitro and in vivo
}

\author{
Yonghong $\mathrm{He}^{\mathrm{a}}$, Da Xing ${ }^{\mathrm{a}, *}$, Guihong Yan ${ }^{\mathrm{a}}$, Ken-ichi Ueda ${ }^{\mathrm{b}}$ \\ ${ }^{a}$ Institute of Laser Life Science, South China Normal University, Guangzhou 510631, China \\ ${ }^{\mathrm{b}}$ Institute of Laser Science, University of Electro-Communications, 1-5-1 Chofugaoka, Chofu, Tokyo 182-8585, Japan
}

Received 24 September 2001; received in revised form 25 January 2002; accepted 28 January 2002

\begin{abstract}
In this work, the chemiluminescence method was engaged for the first time to detect the active oxygen species during sonodynamic action in vitro and in vivo. We used FCLA (3,7-dihydro-6-[4-[2-[ $N^{\prime}$-(5-fluoresceinyl)thioureido]-ethoxy]phenyl]2-methylimidazo[1,2-a]pyrazin-3-one sodium salt), which can selectively react with singlet oxygen $\left({ }^{1} \mathrm{O}_{2}\right)$ or superoxide anion $\left(\mathrm{O}_{2}^{-}\right)$to emit photons, to detect in real time oxygen free radical formation in the sonosensitization of hematoporphyrin derivative. The results show that ${ }^{1} \mathrm{O}_{2}$ is involved in the sonosensitization. In in vivo experiments, a tumor-imaging method by sonodynamic chemiluminescence detection was established. This method could have potential applications in clinics for tumor diagnosis. (C) 2002 Elsevier Science Ireland Ltd. All rights reserved.
\end{abstract}

Keywords: Sonodynamic therapy; Chemiluminescence; Oxygen free radicals

\section{Introduction}

Umemura et al. [1,2] have reported the synergistic effect of ultrasound and hematoporphyrin or galliumporphyrin derivative ATX-70 on tumor treatment. Compared with 'photodynamic therapy', this phenomenon has been termed 'sonodynamic therapy' (SDT) and attracts increasing attention [3-10]. SDT appears to be a promising modality for cancer treatment, for ultrasound can penetrate deep within the

\footnotetext{
* Corresponding author. Tel.: +86-20-8521-0089; fax: +86-208521-6052.

E-mail address: xingda@scnu.edu.cn (D. Xing).

Abbreviations: CL, chemiluminescence; EPR, electron paramagnetic resonance; $\mathrm{HpD}$, hematoporphyrin derivative; FCLA, 3,7dihydro-6-[4-[2-[N'-(5-fluoresceinyl)thioureido]-ethoxy]phenyl]-2methylimidazo[1,2-a]pyrazin-3-one sodium salt; SOD, superoxide anion dismutase; PMT, photomultiplier tube; $\mathrm{CCD}$, charge coupled device; cps, counts per second; ${ }^{1} \mathrm{O}_{2}$, singlet oxygen; $\mathrm{O}_{2}^{-}$, superoxide anion; $\mathrm{NaN}_{3}$, sodium azide; $\mathrm{PBS}$, phosphate-buffered saline
}

tissue and can be focused in a small region of tumor to chemically activate relatively non-toxic molecules thus minimizing undesirable side effects [3]. The mechanism of SDT is still under investigation. Sakusabe et al. [4] reported that the sonosensitizer could increase the anti-tumor effects of ultrasound by increasing the production of singlet oxygen $\left({ }^{1} \mathrm{O}_{2}\right)$ and other oxygen species. Yumita et al. [5] suggested that ultrasonically generated active oxygen plays a primary role in ultrasonically induced cell damage in the presence of photofrin II. However, Miyoshi et al. [6] have shown the evidence against ${ }^{1} \mathrm{O}_{2}$ formation by sonolysis of aqueous oxygen-saturated solutions of hematoporphyrin and rose Bengal by use of electron paramagnetic resonance (EPR) spectra of DRD156. Whether ${ }^{1} \mathrm{O}_{2}$ was involved in the sonosensitization process is still a controversy. In the previous works, the oxygen free radicals were detected by EPR [6-9] after ultrasound irradiation. This non-real-time 
method, which was often combined with the spintrapping technique, is not a direct detection for ${ }^{1} \mathrm{O}_{2}$.

In this work, we used a chemiluminescence (CL) agent, FCLA, which can efficiently react with ${ }^{1} \mathrm{O}_{2}$ or superoxide anion $\left(\mathrm{O}_{2}^{-}\right)$[11-13], to indicate in real time oxygen free radical formation during the sonosensitization both in vitro and in vivo. In in vitro experiments, adding sonosensitizer to air-saturated solution could largely enhance the CL intensity of FCLA. Nitrogen saturation eliminated the CL of FCLA. In in vivo experiments, a tumor-bearing mouse was imaged during sonosensitization with FCLA as the CL probe of ${ }^{1} \mathrm{O}_{2}$ and $\mathrm{O}_{2}^{-}$.

\section{Materials and methods}

\subsection{Equipment}

The experimental apparatus is shown as Fig. 1. The CL light is detected by a photomultiplier tube (PMT) or a cooled charge coupled device (CCD) detector. The PMT (CR-129, Humamatsu, Beijing) is biased at $-900 \mathrm{~V}$ and cooled to $0{ }^{\circ} \mathrm{C}$ to reduce the thermal noise. The signal from the PMT is amplified and discriminated into TTL pulses. The pulses are counted by the LabView software (National Instruments) through an I/O counting card. The CL intensity was represented as counts per second (cps). A back-illuminated, cooled CCD (Princeton Instruments, TE/ CCD-512TKB) detector is used to detect two-dimensional images. The spectra response range of the CCD is from 300 to $800 \mathrm{~nm}$ with the peak at about $500 \mathrm{~nm}$,

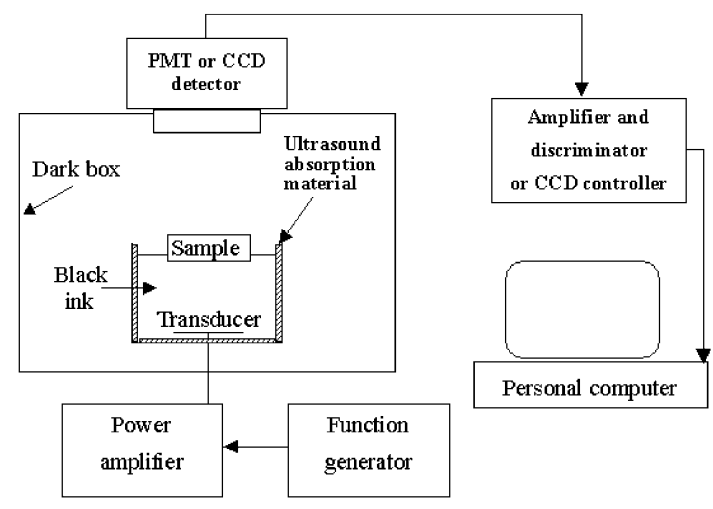

Fig. 1. A schematic diagram of the experimental setup. and the average quantum efficiency is $60 \%$. The CCD surface is cooled to $-58{ }^{\circ} \mathrm{C}$ to reduce the thermal noise down to 0.003 e/pixel s. An ST-130 controller controls data acquisition and transfers the data to a computer in which the digitized image is processed by Winview software.

A function generator (Tektronics, AFG320) produces a $40 \mathrm{kHz}$ sinusoidal signal to a power amplifier (ENI Co. Ltd., 2100L), which amplifies the signal to $50 \mathrm{~W}$ to drive the ultrasonic transducer $(5 \mathrm{~cm}$ in diameter, Meza Guangzhou, China). To reach the sample, the ultrasound passes through distilled water with black ink to avoid the disturbance of the sonoluminescence of the water bath.

\subsection{Chemicals and experimental methods}

FCLA was from Tokyo Kasei Co. Ltd., Japan. Hematoporphyrin derivative (HpD, $100 \mathrm{mg} / 20 \mathrm{ml}$ ) was purchased from Beijing Pharma Institute, China. Superoxide anion dismutase (SOD) was from Sigma. Sodium azide $\left(\mathrm{NaN}_{3}\right.$, analytic purity) was from Guangzhou chemical agent factory, China. To prepare stock solution the agents were dissolved in phosphate-buffered saline (PBS).

In in vitro measurement, the volume of samples was maintained at $2 \mathrm{ml}$ during ultrasound exposure. The final concentration of FCLA, HpD, SOD and $\mathrm{NaN}_{3}$ were $1 \mu \mathrm{mol} / \mathrm{l}, 1 \mu \mathrm{g} / \mathrm{ml}, 1 \mu \mathrm{mol} / \mathrm{l}$ and 2 $\mathrm{mmol} / \mathrm{l}$, respectively. The sample was gas-saturated with air or nitrogen bubbling.

In in vivo experiments, a male nude mouse (BALB/ c-nu/nu) was used to establish transplanted cancer. The human SWO-38 cell line was established from grade $1-11$ glioma tumor. Cell suspension $\left(4 \times 10^{6}\right.$ cells in $0.2 \mathrm{ml}$ PBS) was injected subcutaneously into the neck of the mouse. The volume of the tumor reached approximately $1000 \mathrm{~mm}^{3} 20$ days after injection.

$\mathrm{HpD}(1 \mathrm{mg} / \mathrm{ml}$ in saline) was injected i.p. into the tumor-bearing mouse $24 \mathrm{~h}$ before measurement. FCLA was dissolved in saline $(5 \mu \mathrm{mol} / \mathrm{l})$ and injected s.c. into the mouse $30 \mathrm{~min}$ before measurement. The mouse was anesthetized with pentobarbital sodium $(50 \mathrm{mg} / \mathrm{kg}$ i.p.), and then was fixed on a metal mesh. An appropriate amount of degassed water with black ink was poured into the pool to soak the abdomen of the mouse. The time of image acquisition was $15 \mathrm{~min}$. 
The water temperature was maintained at $15{ }^{\circ} \mathrm{C}$ by circulation.

\section{Results and discussion}

The effect of sonodynamic action and free radical scavengers on the chemiluminescent intensity of airand nitrogen-saturated FCLA solution is shown in Fig. 2. The background counts of the PMT detection system are about $45 \mathrm{cps}$ (intensity corresponding to the time 1-4 s). The FCLA solution has a luminescent intensity of $703 \mathrm{cps}(5-10 \mathrm{~s})$ without sonication. When ultrasound is applied, the intensity of FCLA solution increases to about $8430 \mathrm{cps}(11-20 \mathrm{~s})$. The emission further greatly increases to $23410 \mathrm{cps}$ by adding $\mathrm{HpD}(21-30 \mathrm{~s})$. This bright luminescence was inhibited partially by SOD (down to $15837 \mathrm{cps}$ during 31-40 s) and partially by $\mathrm{NaN}_{3}$ (further down to $2330 \mathrm{cps}$ during $41-50 \mathrm{~s}$ ). Since the active oxygen species formation depends on oxygen, we investigated the effect of $\mathrm{N}_{2}$-saturated condition on the CL intensity of the solutions. Under the $\mathrm{N}_{2}$-saturating condition, the CL intensity of both FCLA and FCLA + HpD solutions was 2840 cps, significantly lower than that under the air-saturating condition.

Ultrasonic irradiation of liquids generates acoustic cavitation: the formation, growth, and collapse of the bubbles in a liquid creates intense local heating which can drive high-energy chemical reactions [14]. Specifically, the sonolysis of water produces $\mathrm{OH}$ and $\mathrm{H}$

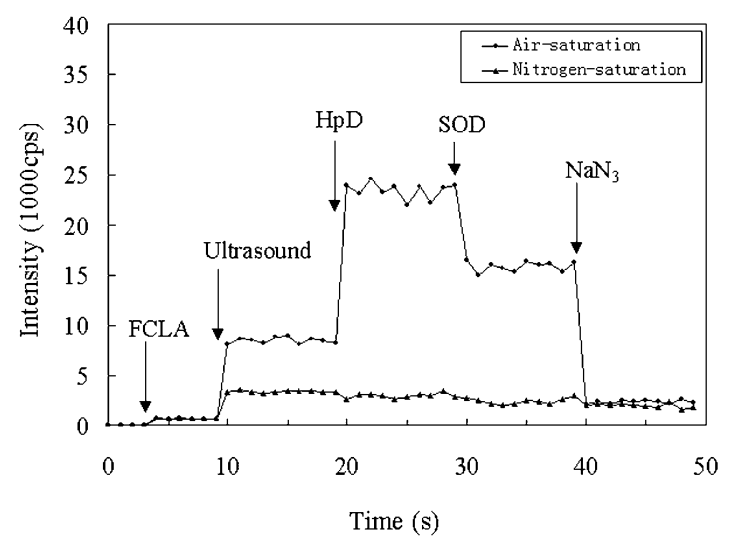

Fig. 2. Effect of sonodynamic action and free radical scavengers on the chemiluminescent intensity of air- and nitrogen-saturated FCLA solution.
[15]. In the presence of $\mathrm{O}_{2}, \mathrm{OH}$ and $\mathrm{H}$ further react with $\mathrm{O}_{2}$ to form active oxygen species, which could react with FCLA to produce the CL. This is the sonochemiluminescence of FCLA as shown in Fig. 2 (the curve under air-saturation condition, phase 11-20 s).

Since FCLA selectively reacts with ${ }^{1} \mathrm{O}_{2}$ or $\mathrm{O}_{2}^{-}$to emit photons, from the enhancement of the $\mathrm{CL}$ of FCLA solution by sonosensitizer HpD under ultrasound (from 8430 to $23410 \mathrm{cps}$ shown in Fig. 2), one could expect that the amount of ${ }^{1} \mathrm{O}_{2}$ and/or $\mathrm{O}_{2}^{-}$ largely increased during sonication. Because the $\mathrm{CL}$ was inhibited by $\mathrm{NaN}_{3}$, a quencher of ${ }^{1} \mathrm{O}_{2}$, and was not completely inhibited by SOD, ${ }^{1} \mathrm{O}_{2}$ was considered to be produced in the sonodynamic action.

To further testify the FCLA CL enhancement by sonosensitization of $\mathrm{HpD}$, the $\mathrm{CCD}$ detector was used to image the two-dimensional CL intensity. Fig. 3 shows CL images of air-saturated FCLA and FCLA + HpD solutions under ultrasound exposure detected by the CCD. The average CL intensity of FCLA + HpD solution (20600 counts/pixel) is much higher than that of FCLA solution (6400 counts/pixel). Both images in Fig. 3 show an excellent uniformity of CL intensity, which indicates that the uniformity of the ultrasound field is well. Fig. 4B shows and a CL image of a tumor-bearing nude mouse under the real-time excitation of the uniform ultrasound field, while Fig. 4A is a photograph of the same mouse for position comparison. It is shown that the $\mathrm{CL}$ emission from tumor region is much stronger than that from other regions. The outline of the tumor is quite clear. After the background subtraction, the average intensity of the emission from the tumor region reaches 6680 counts/pixel, while that from other regions is only 1560 counts/pixel. FCLA diffused to tissues around the cross (including the tumor) within several minutes after it was injected subcutaneously. HpD could accumulate in tumor tissues after it was injected into the body of the mouse $24 \mathrm{~h}$ before. So the emission from tumor tissue could be sonodynamic CL of FCLA, while the light from other regions is the sonoluminescence of tissues.

\section{Conclusions}

The oxygen free radicals formation during sonication process was detected in real time and directly 


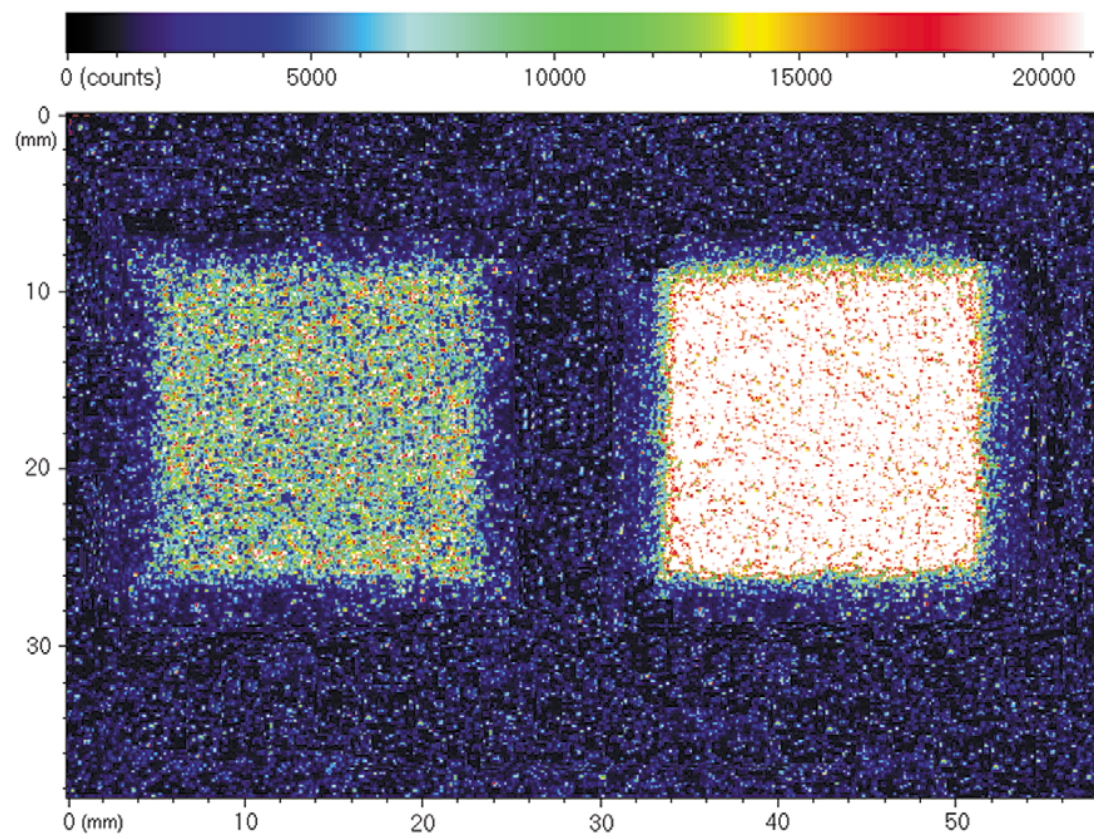

Fig. 3. The chemiluminescence image of FCLA (in the left cup) and FCLA + HpD (in the right cup) solution under ultrasound exposure.

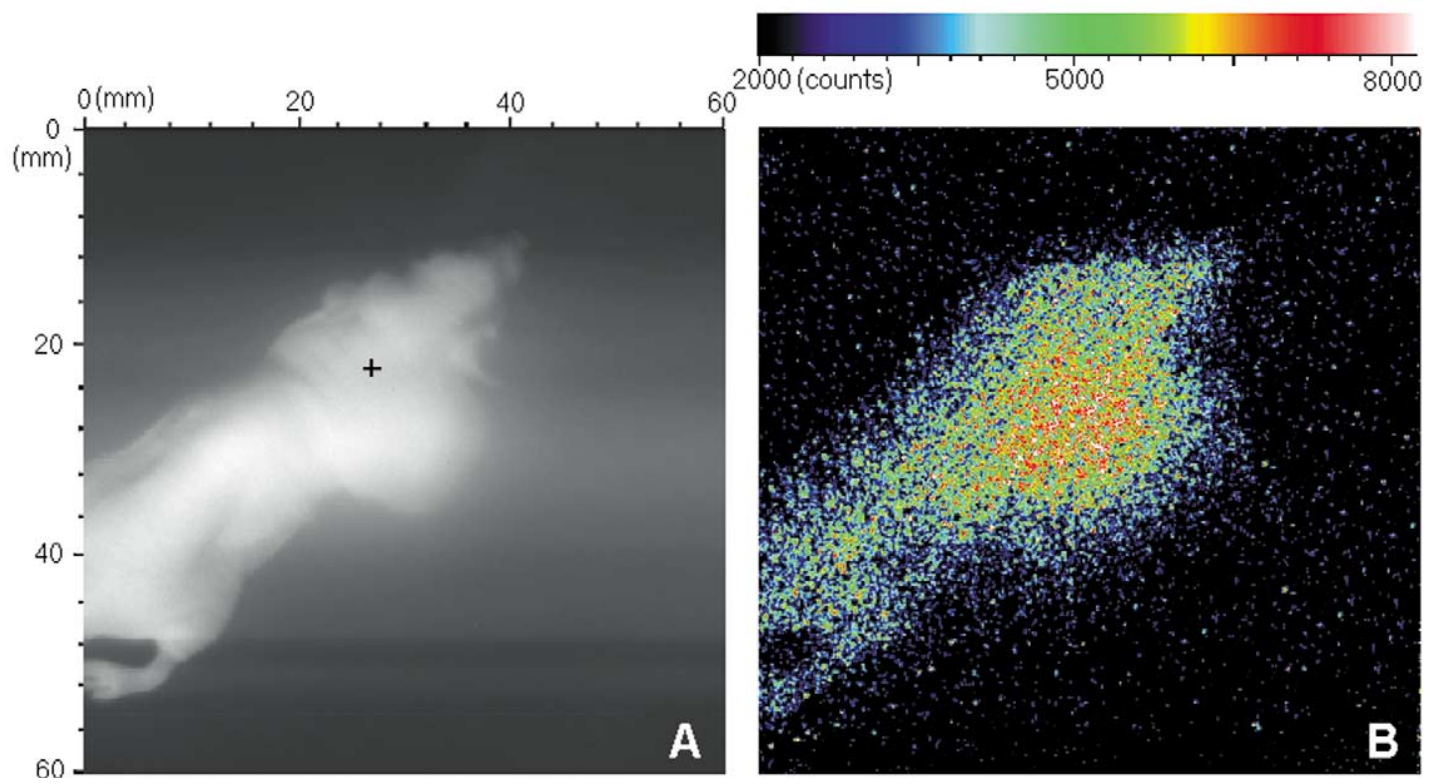

Fig. 4. (A) Photograph of the tumor-bearing nude mouse. (B) FCLA chemiluminescence image of the same mouse under sonodynamic action. Sonosensitizer HpD was injected i.p. 24 h before, FCLA was injected subcutaneously at the cross position 30 min before. 
with $\mathrm{CL}$ method. ${ }^{1} \mathrm{O}_{2}$ and $\mathrm{O}_{2}^{-}$were largely produced during sonodynamic action. In in vivo experiments, a tumor-imaging method by sonodynamic chemiluminescence detection was established. This method could have potential applications in clinics for tumor diagnosis.

\section{Acknowledgements}

This research was supported by the National Science Fund for Distinguished Young Scholars of China (No. 69725009), and Team Project of the Natural Science Foundation of Guangdong Province (No. 015012).

\section{References}

[1] N. Yumita, R. Nishigaki, K. Umemura, S. Umemura, Hematoporphyrin and sensitizer of cell-damaging effect of ultrasound, Jpn. J. Cancer Res. 80 (1989) 219-222.

[2] S. Umemura, N. Yumita, R. Nishigaki, Enhancement of ultrasonically induced cell damage by gallium-porphyrin complex ATX-70, Jpn. J. Cancer Res. 84 (1993) 582-588.

[3] V. Misik, P. Riesz, Free radical intermediates in sonodynamic therapy, Ann. N.Y. Acad. Sci. 899 (2000) 335-348.

[4] N. Sakusabe, K. Okada, K. Sato, S. Kamada, Y. Yoshida, T. Suzuki, Enhanced sonodynamic antitumor effect of ultrasound in the presence of nonsteroidal anti-inflammatory drugs, Jpn. J. Cancer Res. 90 (1999) 1146-1151.

[5] N. Yumita, S. Umemura, R. Nishigaki, Ultrasonically induced cell damage enhanced by photofrin II: mechanism of sonodynamic activation, In Vivo 14 (2000) 425-429.
[6] N. Miyoshi, T. Igarashi, P. Riesz, Evidence against singlet oxygen formation by sonolysis of aqueous oxygen-saturated solutions of hematoporphyrin and rose Bengal. The mechanism of sonodynamic therapy, Ultrason. Sonochem. 7 (2000) 121-124.

[7] N. Miyoshi, V. Misik, M. Fukuda, P. Riesz, Effect of galliumporphyrin analogue ATX-70 on nitroxide formation from a cyclic secondary amine by ultrasound: on the mechanism of sonodynamic activation, Radiat. Res. 143 (1995) 194-202.

[8] V. Misik, P. Riesz, Peroxyl radical formation in aqueous solutions of $\mathrm{N}, \mathrm{N}$-dimethylformamide, $\mathrm{N}$-methylformamide, and dimethylsulfoxide by ultrasound: implications for sonosensitized cell killing, Free Radic. Biol. Med. 20 (1996) 129-138.

[9] V. Misik, N. Miyoshi, P. Riesz, EPR spin trapping study of the decomposition of azo compounds in aqueous solutions by ultrasound: potential for use as sonodynamic sensitizers for cell killing, Free Radic. Biol. Med. 25 (1996) 3-22.

[10] P.K. Hristov, L.A. Petrov, E.M. Russanov, Lipid peroxidation induced by ultrasonication in Ehrlich ascitic tumor cells, Cancer Lett. 121 (1997) 7-10.

[11] K. Fujimori, T. Komiyama, H. Tabata, T. Nojima, K. Ishiguro, Y. Sawaki, H. Tatsuzawa, M. Nakano, Chemiluminescence of cypridina luciferin analogs. Part 3. MCLA chemiluminescence with singlet oxygen generated by the retro-diels-alder reaction of a naphthalene endoperoxide, Photochem. Photobiol. 68 (1998) 143-149.

[12] M. Nakano, K. Sugioka, Y. Ushuima, T. Goto, Chemiluminescence probe with cypridina luciferin analog estimating the ability of human granulocytes to generate $\mathrm{O}_{2}^{-}$, Anal. Biochem. 159 (1986) 363-369.

[13] M. Nakano, Detection of active oxygen species in biological systems, Cell. Mol. Neurobiol. 18 (1998) 565-579.

[14] K.S. Suslick, Sonochemistry, Science 247 (1990) 1439-1445.

[15] K. Makino, M.M. Mossoba, P. Riesz, Chemical effects of ultrasound on aqueous solutions: Formation of hydroxyl radicals and hydrogen atoms, J. Phys. Chem. 87 (1983) 1369-1377. 